\title{
Global models for moving contact lines
}

\author{
Javier A. Diez, ${ }^{*}$ L. Kondic, ${ }^{\dagger}$ and Andrea Bertozzi \\ Department of Mathematics and Center for Nonlinear and Complex Systems, Duke University, Durham, North Carolina 27708
}

(Received 26 April 2000; revised manuscript received 20 September 2000; published 22 December 2000)

\begin{abstract}
We consider thin film flows driven by surface tension and gravity. Within the framework of the lubrication approximation, we study the contact line motion using global models where either precursor film or slip are allowed. We show that completely wetting films can be simulated under both conditions without requiring direct tracking of the contact line interface. We perform a comparative study of standard and positivity preserving numerical methods for these problems in one space dimension, with the ultimate goal of choosing the best method applicable to two-dimensional problems. We find a considerable computational advantage of the precursor film model over the slipping models.
\end{abstract}

DOI: 10.1103/PhysRevE.63.011208

PACS number(s): 47.11.+j, 68.15.+e, 47.20.Ma, 47.20.Ky

\section{INTRODUCTION}

The coating of a solid surface by a thin liquid film and, in particular the dynamics of the contact line, is a problem of interest from both practical and theoretical points of view $[1-3]$. This problem is usually approached within the framework of lubrication approximation, where the velocity field is depth-averaged over the thickness of the film. This approach reduces Navier-Stokes equations to a more tractable single fourth order PDE which governs the time evolution of the film thickness $h(x, y, t)$. In the context of fluid spreading on a horizontal substrate this equation, suitably scaled, is given by (e.g., $[4,5])$

$$
\frac{\partial h}{\partial t}+\boldsymbol{\nabla} \cdot\left[D(h) \nabla \nabla^{2} h\right]-\mathcal{G} \boldsymbol{\nabla} \cdot\left[D(h)^{3} \boldsymbol{\nabla} h\right]=0 .
$$

The fourth order term results from the capillary effects, while the lower order terms model the gravitational forces. $\mathcal{G}$ is a constant defined by the scaling (see Sec. VI). Additional terms arising from thermal effects, centrifugal forces, etc., might be included. If one assumes usual no-slip boundary conditions at the substrate, the nonlinear diffusion is given by $D(h)=h^{n}$, with $n=3$. Equations of the type of Eq. (1) are also important in other fields for different values of $n$. Some examples are the evolution of a thin neck in a HeleShaw cell [6], the diffusion of dopants in semiconductors [7,8], Cahn-Hillard models with degenerate mobility [9], population dynamics [10], and problems in plasticity [11].

A major hindrance in the development of the theory for the problem of fluid spreading is the incomplete knowledge of the physics at the contact line, where liquid, gas, and solid phase meet. A moving contact line coupled with a no-slip boundary condition leads to a multivalued fluid velocity at the contact line. As a consequence, the viscous dissipation rate as well as the stresses diverge as $h \rightarrow 0$. This constitutes

\footnotetext{
*Permanent address: Instituto de Física Arroyo Seco, Universidad Nacional del Centro, Pinto 399, 7000, Tandil, Argentina. Electronic address: jdiez@exa.unicen.edu.ar.

${ }^{\dagger}$ Present address: Department of Mathematical Sciences, New Jersey Institute of Technology, Newark, NJ 07102.
}

the contact line paradox $[1,2]$; this phenomenon manifests itself in the lubrication equation, Eq. (1), in that advancing front solutions do not exist for $n \geqslant 3$ [12].

In the general context of partially wetting fluids, the contact line problem is typically addressed by either relaxing no-slip boundary condition, or by accounting for the effects of the long range intermolecular van der Waals forces (disjoining pressure). The former approach leads to introducing a new term in the diffusivity $D(h)$, while the latter adds another nonlinear second order term, both effectively modifying the fluid behavior in the vicinity of the contact line. In the case of completely wetting fluids, experimental evidence $[13,14]$ motivates the inclusion of a microscopic precursor film in front of the apparent contact line, thus removing the singularity. This approach is consistent with a van der Waals model in the case of a favorable disjoining pressure (see, e.g., $[2,15])$.

In this work we concentrate on the case of completely wetting fluids. We propose to formulate a "global" model which considers the contact line as part of a system that includes both the wet and dry parts of the surface. The goal is to have a method that captures changes in topology such as film rupture and merger without explicitly tracking the interface. In this sense, the models are in the same spirit as recent Cahn-Hilliard models introduced to capture topological transitions in fluids $[16,17]$. This direction of research requires direct solution of Eq. (1). The main subject of this paper is to explore computational methods to achieve this goal.

In developing these methods, we first concentrate on the simpler equation which includes only capillary effects, so that $\mathcal{G}=0$ in Eq. (1), and consider the simple power law model $D(h)=h^{n}$

$$
\frac{\partial h}{\partial t}=-\nabla \cdot\left[h^{n} \nabla \nabla^{2} h\right]
$$

This fourth-order diffusion equation is degenerate, since the diffusivity vanishes as $h \rightarrow 0$. It is of interest to consider this equation for different values of the exponent $n(n \neq 3)$, for the following reasons. First, relaxing no slip-boundary condition at the contact line leads to a modified diffusivity in Eq. (1), given now by $D_{s}(h)=h^{3}+\Lambda_{s} h^{s}$, where $\lambda(h)$ $=\Lambda_{s} /\left(3 h^{2-s}\right)$ is the slipping length, and $\Lambda_{s}$ is a positive 
constant. So, the behavior of the diffusivity for small $h$ is modified from $D \sim h^{3}$ to $D \sim h^{s}$. Typically, $s=1$ (singular slip), and $s=2$ (constant slip) have been used [18-23]. The second motivation for analyzing Eq. (2) with $n \neq 3$ is that for $n=1,2$ source type self-similar solutions exist, allowing for direct comparison of the computational and theoretical results.

The equations of the type of Eq. (2) do not satisfy the maximum principle (see, e.g., [24]); as a result positive initial data might lead to a solution which changes sign. However, one remarkable consequence of the nonlinear structure of Eq. (2) is that it does permit non-negative solutions from the non-negative initial data. Still, even for the problems where positivity of the solution is guaranteed analytically for a continuous model, computations might still lead to premature or false singularities $(h \leqslant 0)$. A nonpositive numerical solution, even if it occurs at a single mesh point, introduces artificial instability and inaccuracy into the system. Standard finite-difference schemes do not necessarily preserve positivity of the solution, in particular if the computations are not well resolved. Recently, several schemes have been developed to address this issue [25-27]. In this work, we use the positivity preserving method presented in [25], which is based on the key idea of Lyapunov dissipation. We empirically test this method by comparing the results to self-similar solutions (where applicable), and to the computational results obtained using standard finite-difference discretization.

In Sec. II we explain the mathematical tools to be implemented in the numerical code, whose main features are presented in Sec. III. This finite difference code solves the onedimensional (planar and radial symmetry) version of Eq. (2). In Sec. IV we use the self-similar solution of the spreading of a constant volume "drop" as a benchmark for the code, and perform a parametric study of convergence for $n=1$ and 2, using either standard or special discretizations of the diffusivity $D(h)$. In the case of physical relevance, $n=3$, we employ both the precursor film and the slipping models, and analyze the spreading of a small drop, where the effects of gravity can be ignored. After performing convergence study, we address the problem of the radial drop spreading for long times and look for the asymptotic behavior of the solution (Sec. V). The addition of gravitational forces is considered in Sec. VI, where we study the effects of gravity on the radial drop spreading. Section VII is devoted to the summary and conclusions of the work.

\section{GLOBAL MODELS AND POSITIVITY OF THE SOLUTION}

A growing body of mathematical research (see the review article [24] and references therein) addresses the questions related to the lack of the maximum principle of the solutions of Eq. (2). In particular, the theory of "weak" nonnegative solutions provides a rigorous context for solutions of Eq. (2) with moving contact lines [28].

The dynamics of solutions of the lubrication equation, Eq. (2), can be understood in the context of several known Lyapunov functions. The Lyapunov function $\mathcal{E}(h)$
$=1 / 2 \int|\boldsymbol{\nabla} h| d x$, associated with the surface tension energy in the lubrication limit, satisfies

$$
d \mathcal{E} / d t=-\int D(h)\left|\nabla^{2} h\right|^{2} d x .
$$

Another Lyapunov function, often referred to as an "entropy," is $\int G(h) d x$, satisfying

$$
\frac{d \int G(h) d x}{d t}=-\int\left|\nabla^{2} h\right|^{2} d x,
$$

where $G^{\prime \prime}(h)=1 / D(h)$. The name entropy is motivated by the Hele-Shaw geometry, $D(h)=h$, for which $G(h)$ $=h \log h$. Such entropies and energies have been used to study the physics of viscous topology transitions in HeleShaw flows $[29,30]$, as well as many mathematical properties of weak solutions of the PDE, Eq. (2) (see [24] and references therein).

Note that $G(h) \sim h^{2-n}$ for $D(h)=h^{n}$, and the entropy is a negative power of $h$ for sufficiently large $n$. Using this fact, one can show that in the planar case (one space dimension) positive initial conditions always yield a positive solution $[31,32]$ for sufficiently large $n$. A practical importance of this fact is that one can construct nonnegative zero contact angle solutions of the PDE for relatively small values of $n$ by using a modified version of the diffusivity $D(h)$, lifting of the initial condition, and passing to the limit. This fact has been proved for the planar case $[33,34]$. This procedure, known as regularization, involves replacing nonnegative (e.g., dropshaped) initial film shapes $h(x, 0)$ with an artificially lifted shape $h(x, 0)+b$ ( $b$ is small and does not represent a physical precursor), and replacing the diffusivity $D$ by the modified diffusivity

$$
\widetilde{D}(h)=\frac{D(h) h^{4}}{\varepsilon D(h)+h^{4}},
$$

where $\varepsilon=\varepsilon(b)$ is also small. Note that $\widetilde{D}(h) \rightarrow D(h)$ as $\varepsilon$ $\rightarrow 0$, and also $\widetilde{D}(h) \rightarrow h^{4} / \varepsilon$ as $h \rightarrow 0$ (for $n<4$ ), which guarantees positivity of the regularized solution. In the case of planar symmetry, in order to guarantee a zero contact angle solution in the limit $b \rightarrow 0, b \geqslant \varepsilon^{1 / 2}$ for $2<n<3$ and $b$ $\geqslant \varepsilon^{2 / 5}$ for $0<n<2$. Here, we take

$$
b=\varepsilon^{0.3},
$$

whenever regularization is used.

This paper compares the behavior of new and standard numerical methods for simulating classical problems involving moving contact lines. All of the methods we consider are finite difference schemes; however, the schemes have a parallel setting as finite element methods [25,26]. Our computational domain $0 \leqslant x \leqslant L$ is divided into $N$ cells of size $\Delta x_{i}$ $(0 \leqslant i \leqslant N)$. We focus on problems in planar or radially symmetric geometry, reducing the computation to one spatial dimension. In this way, we can accurately test the performance of the different models and schemes under mesh re- 
finement. We use centered finite differences to approximate the spatial derivatives in Eq. (2). For the planar case, the remaining part of the spatial discretization is the particular choice of numerical diffusivity $D_{i+1 / 2}$ midway between the grid points $x_{i}$ and $x_{i+1}$. The most obvious choice is

$$
D_{i+1 / 2}=\frac{1}{2}\left[D\left(h_{i}\right)+D\left(h_{i+1}\right)\right],
$$

used in $[32,35-37]$ which we call the standard scheme $(\mathcal{S S})$. In fact, this naive interpolation may result in a numerical solution that becomes negative at some finite time even when the PDE is sufficiently degenerate to guarantee positivity [25].

Recently [24,25,38], a positivity preserving scheme $(\mathcal{P P S})$ has been proposed to avoid possible negative values of $h$ for $n \geqslant 2$. This scheme results in a discrete form of the entropy dissipation, Eq. (4), thereby producing a positive solution of the PDE, Eq. (2). For the planar case, the discrete diffusivity is

$$
D_{i+1 / 2}= \begin{cases}\frac{h_{i+1}-h_{i}}{g_{i+1}-g_{i}}, & h_{i+1} \neq h_{i}, \\ D\left(h_{i}\right), & h_{i+1}=h_{i},\end{cases}
$$

where $g(h)$ is given by

$$
g(h)=\int \frac{d h}{D(h)} .
$$

This scheme produces a numerical solution that remains positive if the initial data is positive provided $n \geqslant 2$. For moving contact line solutions when $n<2$ (as in the case of singular slipping; see Sec. V A 2), one can combine the regularization method above with the entropy dissipating numerical scheme to produce a modified scheme that incorporates regularization, lifting, and a discretization of the regularized diffusivity of the form given by Eq. (8). For more details and mathematical proofs about the regularization [32,34] and this scheme see Refs. [25] and [38].

\section{OUTLINE OF THE NUMERICAL METHOD}

We have developed a numerical code to solve Eq. (2) for planar and radial symmetry. Assuming such a geometry, Eq. (2) becomes

$$
\frac{\partial h}{\partial t}+x^{-\alpha} \frac{\partial}{\partial x}\left(x^{\alpha} D(h) \frac{\partial c}{\partial x}\right)=0,
$$

where the curvature $c$ is given by

$$
c=x^{-\alpha} \frac{\partial}{\partial x}\left(x^{\alpha} \frac{\partial h}{\partial x}\right)
$$

and $\alpha=0,1$ stands for planar or radial symmetry, respectively. The second term of Eq. (10) is discretized by using a centered finite difference scheme, so that we get

$$
\frac{d h_{i}}{d t}+f_{i}=0,
$$

where $f_{i}$ depends on the five neighboring grid points as

$$
f_{i}=\sum_{m=-2}^{2} a_{m} h_{i+m}
$$

and the prefactors $a_{m}$ are linear functions of the diffusivities $D_{i \pm 1 / 2} . D_{i \pm 1 / 2}$ contains the nonlinearity of the equation and is given by an appropriate interpolation of $D(h)$. Though the code was developed for nonuniform grids $\Delta x_{i}$, for simplicity, here we make computations only for a constant mesh size $\Delta x$. To enforce the boundary condition, we require two ghost cells on the left $(i=-1,-2)$ and right $(i=N+1$, $N+2)$.

The scheme conserves a discrete form of the volume

$$
\sum_{i=0}^{N}\left(2 \pi x_{i}\right)^{\alpha} h_{i}^{\nu} \Delta x_{i}=\sum_{i=0}^{N}\left(2 \pi x_{i}\right)^{\alpha} h_{i}^{\nu+1} \Delta x_{i},
$$

where the superscript $\nu$ stands for the time $t^{\nu}=t^{\nu-1}+\Delta t^{\nu}$, and $\Delta t^{\nu}$ is the $\nu$ th (variable) time step. Volume conservation at the boundary results from a no-flux condition there, $\phi$ $=D(h) \partial c / \partial x=0$. In addition, we also assume $\partial h / \partial x=0$ there. In the discretization, $\phi$ is centered between grid points, thus $\phi=0$ in the middle of each first fictitious cell.

The coupled system of ODE's, Eq. (12) is discretized in time using the standard method

$$
\frac{h_{i}^{\nu+1}-h_{i}^{\nu}}{\Delta t^{\nu}}+\theta f_{i}^{\nu+1}+(1-\theta) f_{i}^{\nu}=0 \quad(0 \leqslant i \leqslant N),
$$

where $0 \leqslant \theta \leqslant 1$. Here, $\theta=0$ gives the forward Euler scheme [explicit, $O\left(\Delta t^{\nu}\right)$ ], $\theta=1$ the backward Euler scheme [implicit, $\left.O\left(\Delta t^{v}\right)\right]$ and $\theta=1 / 2$ the Crank-Nicholson scheme [implicit, $O\left(\Delta t^{\nu}\right)^{2}$ ]. Equation (15) specifies a system of nonlinear algebraic equations for $h_{i}^{\nu+1}$, which is solved using Newton-Kantorovich method. The solution at time $t^{\nu+1}$ is calculated by requiring that the local error is less than a given tolerance (we use $10^{-10}$ throughout this work).

The time evolution from a given initial condition $h_{i}(0)$ $(0 \leqslant i \leqslant N)$ is performed with $\theta=1 / 2$ and variable time steps, the size of which is limited by two requirements: (a) that the solution is strictly positive everywhere in the domain, and (b) that an accuracy condition is satisfied. The requirement (a) is enforced when the Newton iteration has converged: if the converged solution is negative anywhere in the domain, the time step is reduced, and the calculation is repeated. The condition (b) is enforced by estimating the local relative error $e_{i}$ of the solution $h_{i}^{\nu+1}$. A Taylor expansion around $h_{i}^{\nu}$ leads to

$$
e_{i}=\frac{\left(\Delta t^{\nu}\right)^{2}}{h_{i}^{\nu}} \frac{d^{2} h_{i}^{\nu}}{d t^{2}}
$$

so that 


$$
e_{i} \approx \frac{2 \Delta t^{\nu}}{\Delta t^{\nu-1}} \frac{\Delta t^{\nu-1} h_{i}^{\nu+1}+\Delta t^{\nu} h_{i}^{\nu-1}-\left(\Delta t^{\nu-1}+\Delta t^{\nu}\right) h_{i}^{\nu}}{\left(\Delta t^{\nu-1}+\Delta t^{\nu}\right) h_{i}^{\nu}}
$$

If $E=\max \left(e_{i}\right)(0 \leqslant i \leqslant N)$ is less than a given upper bound $E^{m}$ (typically, $E^{m}=10^{-2}-10^{-3}$ ), the solution $h_{i}^{\nu+1}$ obtained with the time step $\Delta t^{\nu}$ is accepted; otherwise, $\Delta t^{\nu}$ is reduced and a new calculation of $h_{i}^{\nu+1}$ is performed. This is done as many times as necessary in order to get $E<E^{m}$. If the application of the conditions (a) and (b) leads to extremely small $\Delta t^{\nu}$, the simulation stops and is deemed unsuccessful. As we illustrate below (see Sec. IV B), this can happen on coarse grids with certain choices of discretization of the diffusivity.

\section{TEST PROBLEM: “DROP” SPREADING}

Before considering the full physical problem of a spreading drop, we perform some numerical tests on a simpler problem with known exact solutions. In both the planar and radial geometries, Eq. (10) with $D(h)=h^{n}$ has a self-similar source-type solution for $n<3$ [39-41]. We use this fact as a benchmark to test performance of the numerical method described above. We also note that the careful analysis of the (unphysical) cases $n=1,2$ will prove very useful in understanding better the performance of the slipping models introduced later in Sec. V.

\section{A. Self-similar solution}

For any constant volume $V$, there exists a unique exact solution of Eq. (10) with compact support, zero contact angle, and the following self-similar structure:

$$
h(x, t)=A t^{\beta} H(\eta),
$$

where $\eta=x / x_{f}$ is the similarity variable,

$$
x_{f}(t)=q t^{\delta}
$$

is the front position, $A$ is a constant determined by the volume $V$, and $q=A^{n / 4}$. The exponents $\delta=1 /(4+(\alpha+1) n)$ and $\beta=(4 \delta-1) / n$ are fixed by dimensional analysis using volume conservation. This similarity solution is called "sourcetype" because it starts as a $\delta$-function with volume $V$, analogous to the Gaussian heat kernel for the heat equation. Plugging this ansatz into Eq. (10) shows that $H$ satisfies the similarity ODE

$$
\beta \eta H-\delta \eta^{2} H^{\prime}+\eta^{-\alpha}\left(\eta^{\alpha} H^{n} C^{\prime}\right)^{\prime}=0,
$$

where $C=\eta^{-\alpha}\left(\eta^{\alpha} H^{\prime}\right)^{\prime}$, and the primes indicate derivative with respect to $\eta$. Volume $V$ and $q$ are related by

$$
V=\int_{0}^{x_{f}}(2 \pi x)^{\alpha} h(x, t) d x=q^{1 / n} \delta I,
$$

where $I=\int_{0}^{1}(2 \pi \eta)^{\alpha} H(\eta) d \eta$ is a shape factor. Equation (20) may be integrated once, leading to

$$
C^{\prime}=\delta \eta H^{1-n},
$$

since $C^{\prime}(0)=0$ by symmetry. This third order equation has boundary conditions:

$$
H^{\prime}(0)=0, \quad H(1)=H^{\prime}(1)=0 .
$$

As mentioned in the Introduction, Eq. (22) can only be solved for $n<3$. The case $n=3$, relevant to physical drops, is discussed in Sec. V.

\section{B. Comparison of numerical and self-similar results}

Here we solve numerically the PDE, Eq. (10), as explained in Sec. III and compare the results with the similarity solutions. We study in detail the computational performance of different interpolation schemes for the diffusivity $(\mathcal{S S}$ and $\mathcal{P P S}$ ). Regularization is added for $n<2$ (see Sec. II). For simplicity, we take the planar drop spreading $(\alpha=0)$ configuration as a test problem for $n=1$ and 2 .

In our computational simulations of Eq. (10), we use the initial condition as given by the self-similar shape $H(\eta)$. Thus, including the "lifting" $b$, we have

$$
h(x, 0)= \begin{cases}H(x) / H_{0}+b, & 0 \leqslant x \leqslant 1, \\ b, & x>1,\end{cases}
$$

where $H_{0}=H(0)$. We compare the numerical values of the solution at the center, $h_{0}(t)$, and the radius $x_{f}(t)$ (apparent contact line) with the theoretically predicted values

$$
x_{f}(t)=h_{0}(t)^{-1}=\left(1+q^{1 / \delta} t\right)^{\delta} .
$$

In order to compare the different schemes explained above, we calculate the evolution up to a given time $\tilde{t}$ using a bounded $\Delta t^{\nu}\left(\leqslant \Delta t_{\max }\right)$ for a given sequence of uniform grids. In all the calculations, $\Delta t_{\max }$ has been chosen small enough so that the dominant source of error is due to the spatial discretization.

\section{The case $n=1$}

For $n=1$, the ODE, Eq. (22) admits the analytical solution

$$
H=H_{0}\left(1-\eta^{2}\right)^{2},
$$

with $\delta=1 / 5, H_{0}=1 / 120, I=8 H_{0} / 15$ for $\alpha=0$, and $\delta=1 / 6$, $H_{0}=1 / 192, I=\pi H_{0} / 3$ for $\alpha=1$.

Figure 1 gives the numerical results for longer times, as well as a comparison with the self-similar solution. The difference between $h(x, t) / h_{0}(t)$ and $H(\eta)$ is very small (less than 0.005 for the time range shown in Fig. 1), thus confirming the ability of our computational method to very closely reproduce the expected self-similar evolution.

We now show that the standard scheme $\mathcal{S S}$ has limited utility since the numerical solutions may become negative on insufficiently fine grids. Figure 2 shows the profiles at $\tilde{t}$ $=0.02\left(\Delta t_{\max }=10^{-6}\right)$ of a sequence of calculations with several $\Delta x$ 's. We use $\mathcal{S S}$ to interpolate the diffusivity $D_{i+1 / 2}$ [see Eq. (7)] and $b=10^{-2}$. Note that the profile has not changed its shape (self-similarity), and it is still well fitted by Eq. (26) with appropriate values of $h_{0}$ and $x_{f}$. The values of 

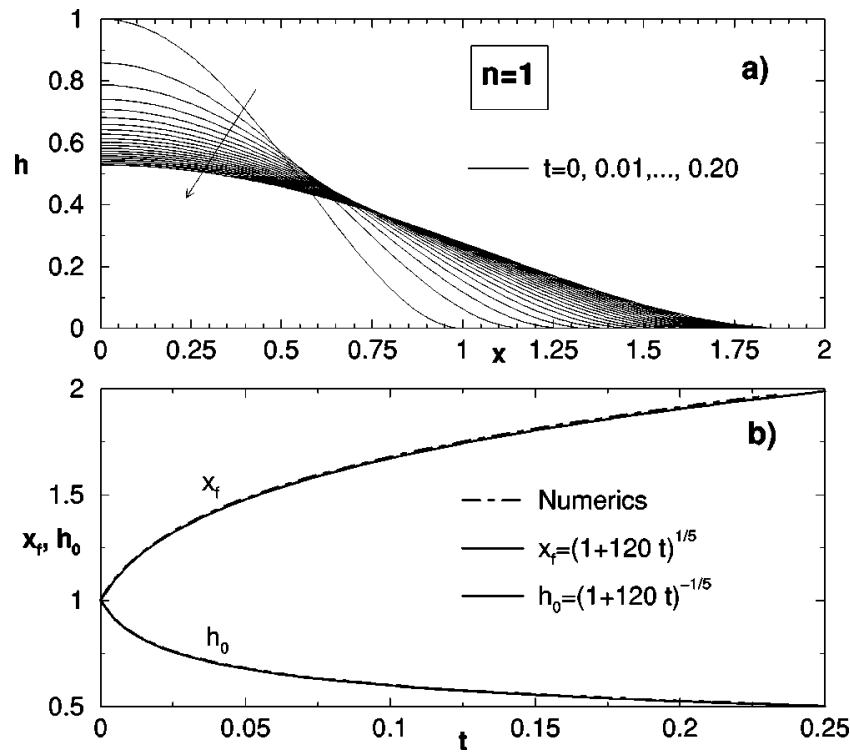

FIG. 1. Self-similarity of the numerical time evolution for $n$ $=1$ and $\alpha=0\left(\Delta x=0.01, b=10^{-4}\right)$, under $\mathcal{P P S}$ with regularization. (a) Thickness profile $h(x, t)$ (the arrow shows the direction of time increase); (b) $x_{f}(t), h_{0}(t)$ compared with the analytical solutions given by Eq. (25), where $q$ is obtained from Eq. (21) with $V$ $=8 / 15$.

$h_{0}$ versus $\Delta x$ are plotted in Fig. 3 (up triangles). It can be seen that for $b=10^{-2}$, the solution does not tend to the exact value $h_{0}(\tilde{t})=\widetilde{h}_{0} \cong 0.78289$ (black dot in Fig. 3). Computations for smaller $b$, such as $10^{-3}$, produce negative values of $h$ in the front region for the same range of $\Delta x$ 's and, therefore, are not valid solutions. One way to try to avoid such negative values is to decrease $\Delta t^{\nu}$, but this procedure leads to $\Delta t^{\nu} \rightarrow 0$. Another possibility is to reduce $\Delta x$ even more, but this is computationally too expensive, and so other options must be explored, such as regularization [see Eq. (5)]. It is seen in Fig. 3 that the addition of regularization for $b$

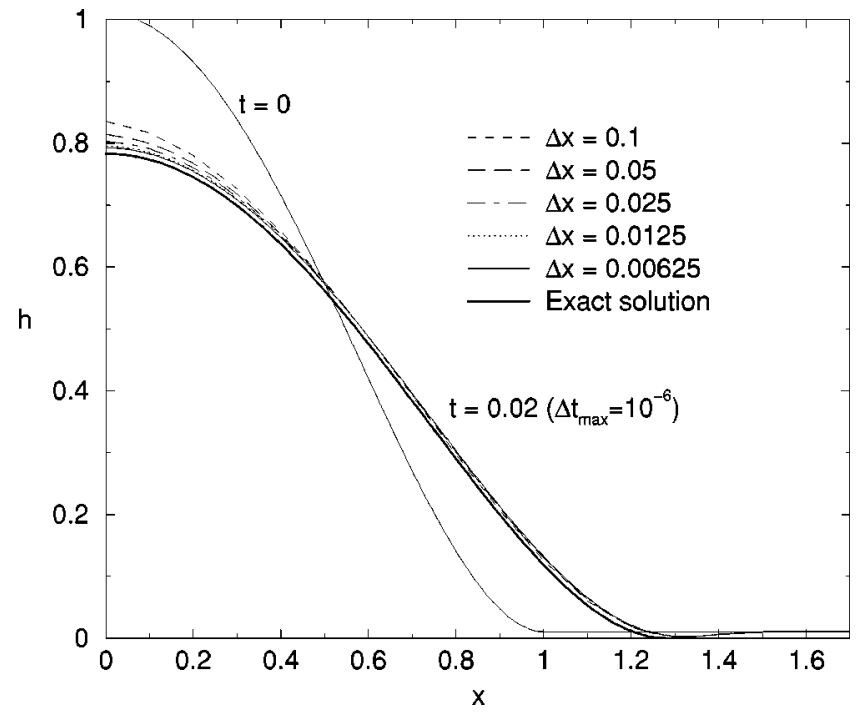

FIG. 2. Planar drop profile for $n=1$ and $b=10^{-2}$ at $t=0.02$ $\left(\Delta t \leqslant 10^{-6}\right)$, using $\mathcal{S S}$ and different $\Delta x$ 's.

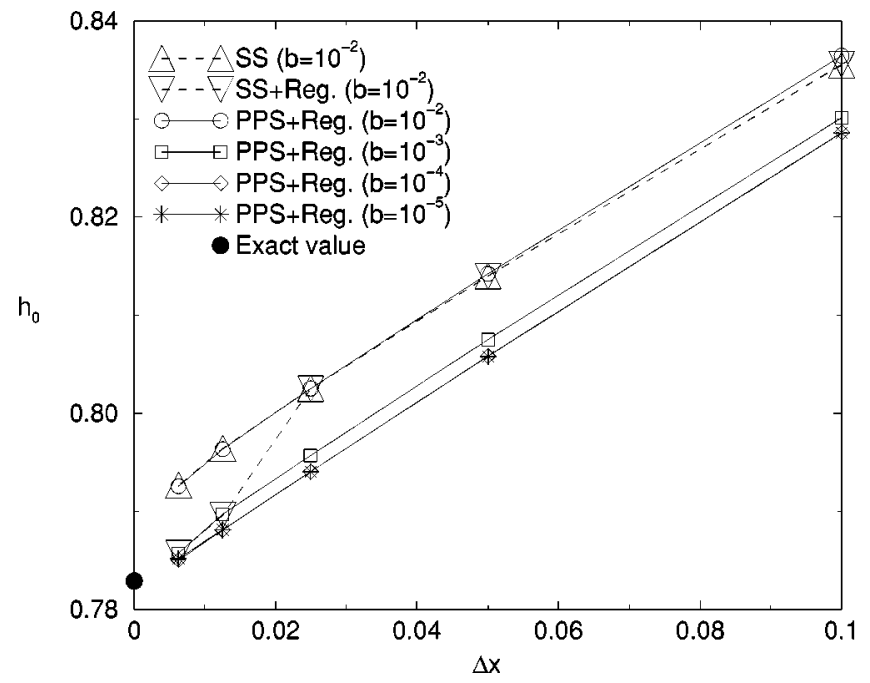

FIG. 3. Thickness $h_{0}$ of the planar drop for $n=1(t=0.02)$ as a function of $\Delta x$ with several values of $b$, under different schemes. For $b=10^{-3}, \mathcal{S S}$ leads to negative results for the film thickness (not shown).

$=10^{-2}$ (down triangles) modifies the behavior for small $\Delta x$, bringing the results closer to the exact value. However, we have found that for $b=10^{-3}$, the regularization is not enough to avoid negativity of the solution.

$\mathcal{P P S}$ removes this problem. Figure 3 shows the results using $\mathcal{P P S}$ plus regularization for $b=10^{-2}-10^{-5}$. Within this scheme, the solutions converge to the exact solution as $\Delta x \rightarrow 0$ for sufficiently small $b$ 's. Figure 4 shows the results as $b \rightarrow 0$ for several $\Delta x$ 's. The solution is practically $b$-independent provided $b<10^{-4}$. We note that without regularization, these calculations cannot be performed since negative values of $h$ do appear using only $\mathcal{P} \mathcal{P S}$ (positivity is not guaranteed for $n \leqslant 2$ ).

It turns out that the solution for $n=1$ can be safely computed only by using $\mathcal{P P S}$ plus regularization, and that $\mathcal{S} \mathcal{S}$ does not work properly in this range of (reasonably small)

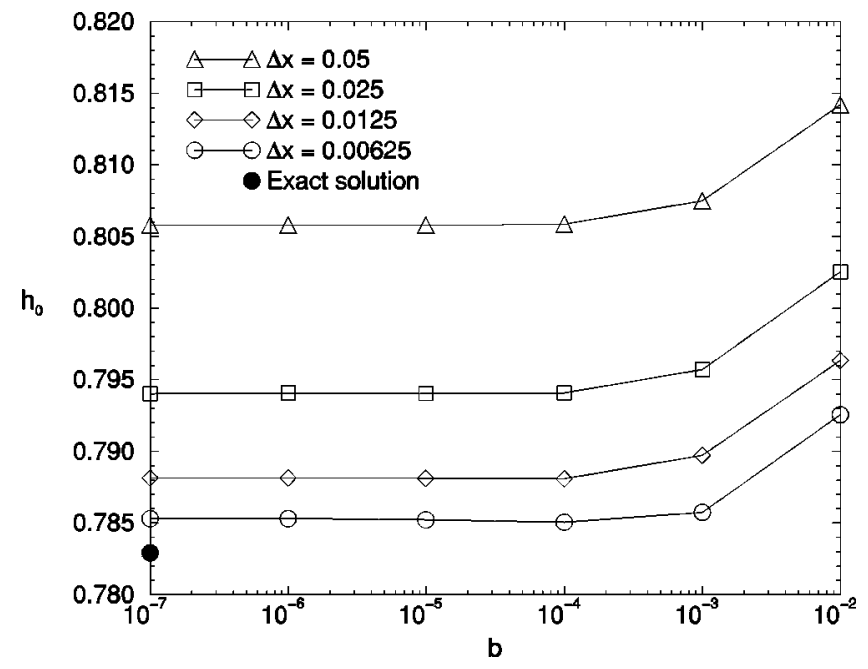

FIG. 4. Thickness $h_{0}$ of the planar drop for $n=1(t=0.02)$ as a function of $b$, using $\mathcal{P P S}$ plus regularization. 


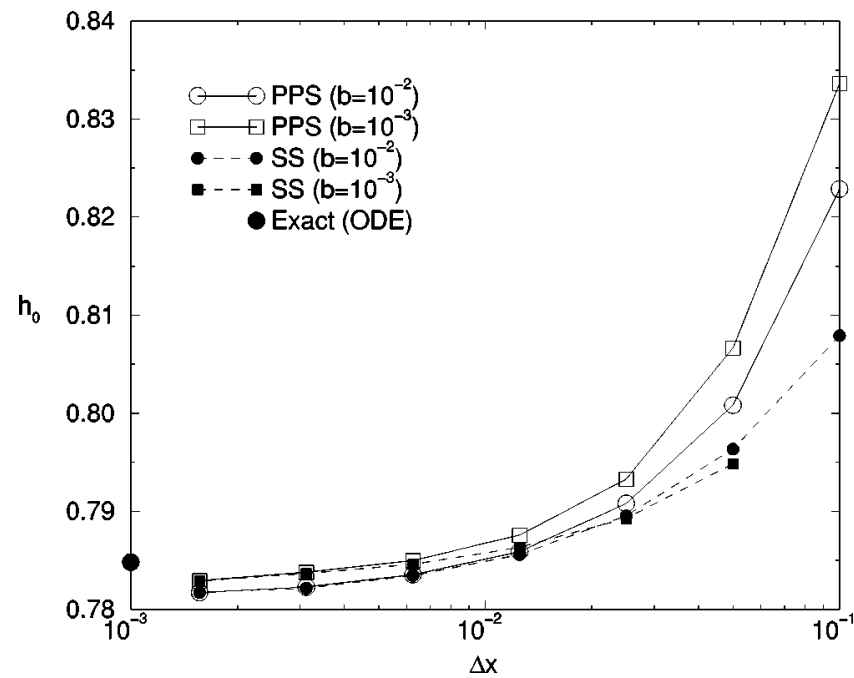

FIG. 5. Thickness $h_{0}$ of the planar drop for $n=2(t=0.1)$ as a function of $\Delta x$ with several values of $b$, under $\mathcal{P P S}$ and $\mathcal{S S}$.

$\Delta x$. Similar result was reported in Ref. [38], where the case $n=1 / 2$ was explored (see also $[26,27]$ for alternative methods).

\section{The case $n=2$}

The case $n=2$ is distinguished by the fact that it is not possible to obtain an analytical solution. Consequently, we numerically solve the ODE (22) using a shooting method. Due to the singularity of $C^{\prime}$ when applying the boundary condition $H(1)=0$ [see Eqs. (22) and (23)], we perform the calculations for decreasingly small values of $H(1)$, and explore the limit $H(1) \rightarrow 0$. The computations show convergence of the solution in this limit, yielding the self-similar profile $H(\eta)$, as well as the coefficient for Eq. (25), in which the exponent $\delta=1 / 6$. We note that varying $\alpha$ (consequently, the geometry of the flow), does not modify the shape of $H(\eta)$ [see Eq. (22)], but it affects the value of $H_{0}$ (consequently, $q$ ). Similarly to the case $n=1$, the numerical solution of the PDE, Eq. (10), successfully reproduces the self similar solution (we skip details for brevity). In what follows we concentrate on comparing the performances of $\mathcal{S S}$ and PPS.

Figure 5 shows that for a given $b$ the results of both schemes approach each other as $\Delta x \rightarrow 0$. SS fails to give a positive solution in the case $b=10^{-3}$ with $\Delta x=0.1\left(\Delta t^{\nu}\right.$ $\rightarrow 0$ ). Note that due to the nonzero $b$, the numerical results do not agree exactly with the solution of Eq. (22) $\left[h_{0}(\tilde{t})\right.$ $\left.=\widetilde{h}_{0} \cong 0.78482\right]$ as $\Delta x \rightarrow 0$, even though the difference decreases for smaller $b$ 's.

In Fig. 6 we show $h_{0}$ as a function of $b$ for several $\Delta x$ 's using $\mathcal{P P S}$ ( $S \mathcal{S}$ gives very similar results). We immediately observe that the convergence is lacking in the range of $\Delta x$ 's for which the case $n=1$ converged, consistent with the results reported in Ref. [38]. One needs to take much smaller $\Delta x$ 's in order to reach convergence; from Fig. 6 we observe that for $\Delta x$ smaller than a certain upper threshold $\left(\cong 3 \times 10^{-3}\right)$, the convergence is reached as $b \rightarrow 0$. This re-

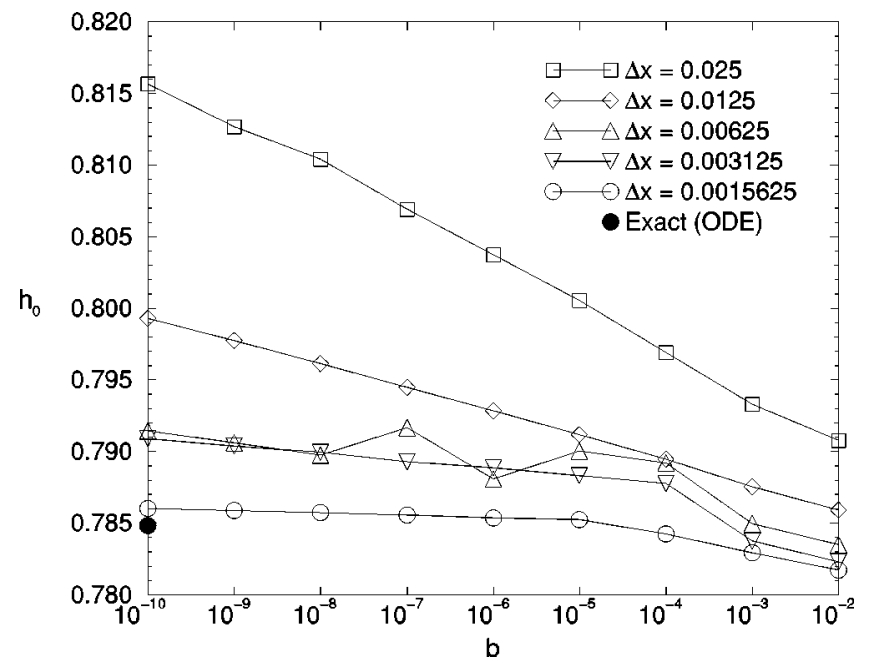

FIG. 6. Thickness $h_{0}$ of the planar drop for $n=2(t=0.1)$ as a function of $b$, using $\mathcal{P P S}$.

quirement on $\Delta x$ is a severe restriction on the simulations for $n=2$ either with $\mathcal{S S}$ or $\mathcal{P P S}$, and will be helpful in understanding poor computational performance of one of the slip models introduced in the next section for the (physical) case $n=3$.

\section{PHYSICAL PROBLEM: SPREADING OF A DROPLET}

The problems presented in the previous section allowed for detailed testing of the performance of our computational methods. Next, we use these methods to simulate a spreading droplet $(n=3)$; we analyze the early time evolution for the planar drop $(\alpha=0)$ and the long time evolution for the radially symmetric drop $(\alpha=1)$.

In the bulk of the drop, the leading order dynamics is governed by Eq. (2) with $n=3$, corresponding to the no-slip boundary condition on the solid surface. There has been much discussion in the literature on the connection between theory, experiment, and the role of Eq. (2) in the bulk $[22,42-45]$. The existence of similarity solutions to Eq. (2) was first considered in [45], where it was discovered that for $n=3$ no solutions exist satisfying the boundary condition $H(1)=0$. This result was later proved rigorously in [39]. In [42] it was suggested that similarity solutions satisfying the boundary condition

$$
H^{\prime}(0)=0, \quad H(1)=b, \quad H^{\prime}(1)=0,
$$

with small but nonzero $b$ are relevant for the dynamics of spreading drops, and also shown that such solutions are linearly stable to perturbations in the bulk. Other authors have argued that the contact line controls the rate of spreading (especially in partial wetting) $[22,44]$ so that a constitutive law is required at the contact line and a quasistatic solution of the PDE results in the bulk. In fact, these two points of view are consistent for the bulk dynamics of the spreading drop problem. To show this, we first present a simple asymptotic argument showing that for very small values of $b$, the similarity solutions satisfying Eq. (27) are closely ap- 

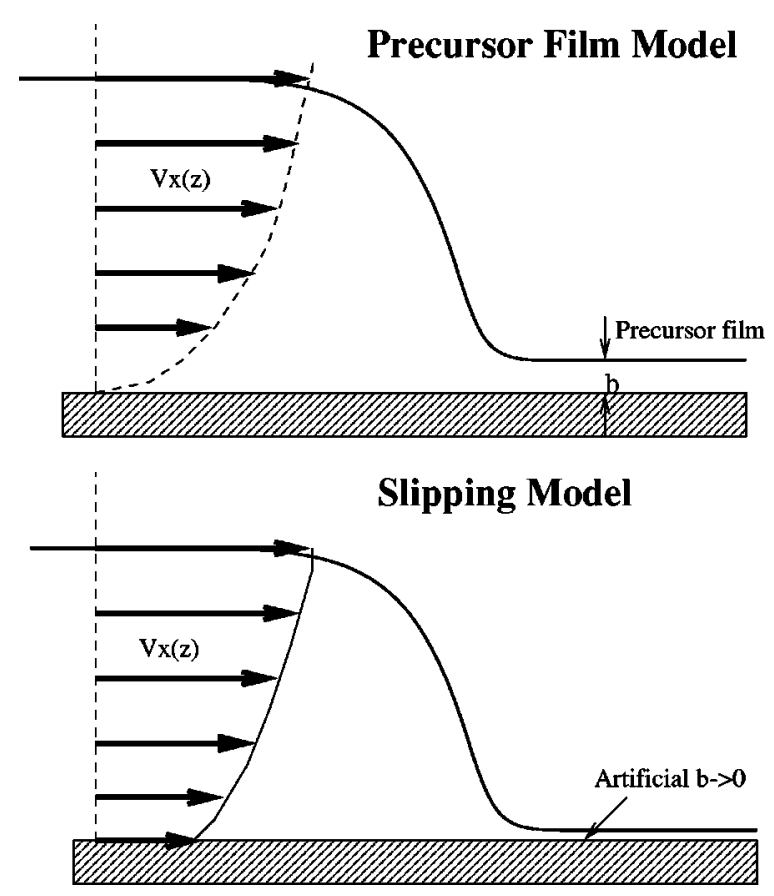

FIG. 7. Sketch of the fluid profile near the contact line, and of the parabolic velocity $v_{x}(z)$ in the bulk for (a) the precursor film model and (b) the slipping models. Note that in (b) $v_{x}(z)$ is not zero at the substrate (slipping), and also that the artificial "lifting'" $b$ must tend to zero.

proximated by the spherical cap solution. This is confirmed by numerical simulations of the full PDE. The main difference between the spherical cap (quasistatic) solutions and the similarity solution occurs in the vicinity of the contact line. This is a result of different contact line dynamics which could lead to different spreading laws.

We now consider the behavior of solutions to Eq. (10) with Eq. (27) in the small $b$ limit. The reader can check that as $b \rightarrow 0$, the solution has $H_{0} \rightarrow \infty$. Thus there is a natural rescaling of the similarity ODE in this limit. Let $\widetilde{H}=H / H_{0}$ so that the similarity ODE, Eq. (22), transforms into the problem

$$
\frac{1}{H_{0}^{3}} \frac{\eta}{10}=\widetilde{H}^{2}\left(\widetilde{H}^{\prime \prime}+\frac{\widetilde{H}^{\prime}}{\eta}\right)^{\prime}
$$

when $\alpha=1$. For large values of $H_{0}$ the solution can be expanded in powers of $H_{0}^{3}$ as $\widetilde{H}=\frac{1}{2}\left(1-\eta^{2}\right)+O\left(H_{0}^{-3}\right)$. The leading order shape is the spherical cap, a steady state solution of the original PDE.

Next, we compare numerical simulations of the full PDE with the similarity solution theory. In Sec. V A we analyze the problem for early times. The computations are performed with several $\Delta x$ 's, and stopped at $\tilde{t}=2\left(\Delta t_{\max }=10^{-4}\right)$. Long time evolution is presented in Sec. V B. For simplicity, in all simulations we begin with the initial condition given by Eqs. (24) and (26). Note that this is not a similarity profile for the droplet equation; we wish to observe the natural dynamics from a generic initial drop shape.

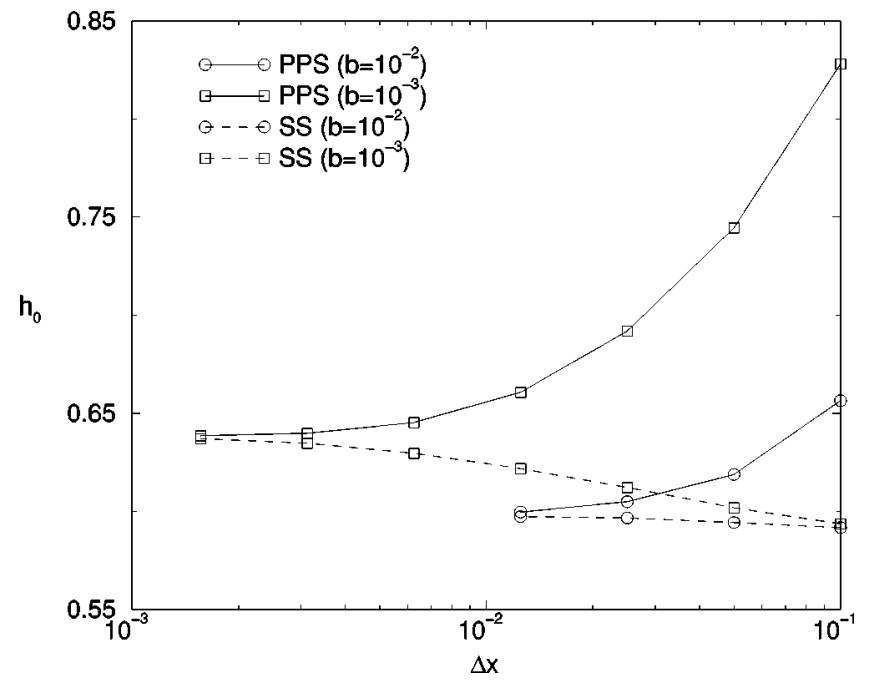

FIG. 8. Thickness $h_{0}$ of the planar drop for $n=3(t=2)$ as a function of $\Delta x$ with several values of the film thickness $b$ (precursor film model), under $\mathcal{P P S}$ and $\mathcal{S S}$.

\section{A. Early time planar evolution}

In order to circumvent the contact line paradox, we employ two different approaches: (a) the precursor film model, and (b) the slipping models. We will see that the computational performance strongly depends on the choice of the model.

\section{Precursor film model}

This model assumes the presence of a true precursor film ahead of the moving contact line, as it has been detected in the experiments $[2,46]$. In this case, the fluid film of thickness $b$ defined before as a numerical artifact has now a physical meaning [see Fig. 7(a)].

The drop profiles (closely resembling a spherical cap) are similar to the case $n=1$. Figure 8 shows the convergence properties with $\mathcal{P P S}$ and $\mathcal{S S}$. Smaller $b$ 's yield greater values of $h_{0}$; this is because the viscous dissipation rate increases for decreasing $b$. Both schemes approach the same value $\tilde{h}_{0}(b)$ for a given $b$, and they demand $\Delta x \leqslant b$ for convergence, consistent with the computations using a slip model [23]. Additional computations using ever smaller $\Delta x$ show that typically an order of magnitude smaller $\Delta x$ is needed for convergence when $\mathcal{S S}$ is used, compared to $\mathcal{P P S}$. It is also interesting to note that, even for large $\Delta x$, we do not encounter the problem of loss of positivity; both schemes can be safely used to compute a positive solution for $n=3$.

We note that, since $b$ accounts for a real precursor film, the requirement $\Delta x \leqslant b$ may be very severe if one intends to describe a very thick drop, but it is not so demanding for thin ones. For instance, for a precursor film of thickness $h_{p}$ $=10 \AA$, we should have $b=10^{-6}$ for a thick drop of height $1 \mathrm{~mm}$. Instead, for much smaller drops (or thin films) of height $1 \mu \mathrm{m}$ and the same precursor film, $b=10^{-3}$ would be appropriate.

\section{Slipping models}

Instead of a precursor model we now consider relaxing the no-slip boundary condition as $[18,20-22]$ 


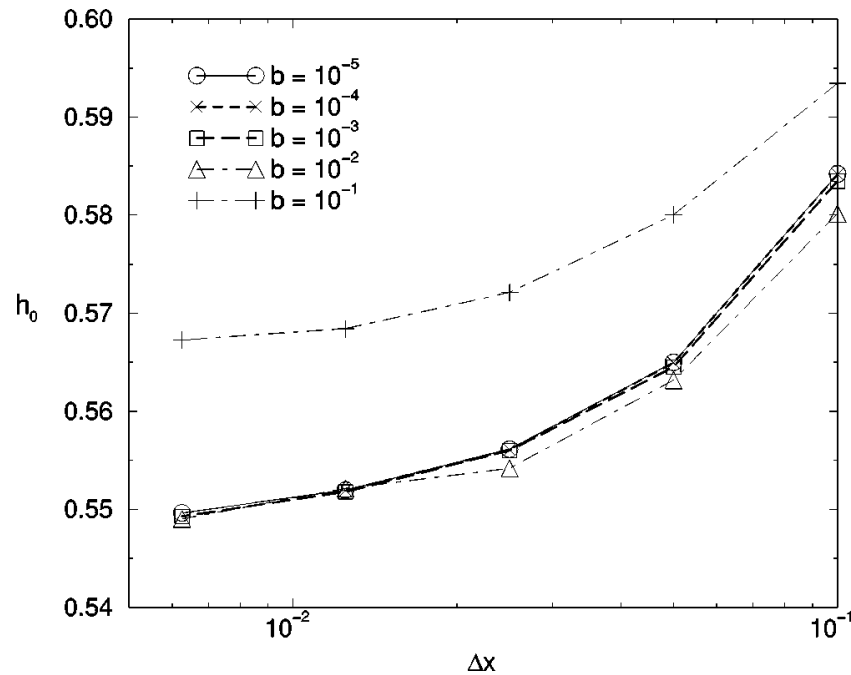

FIG. 9. Thickness $h_{0}$ of the planar drop for $n=3(t=2)$ as a function of $\Delta x$ with several values of the artificial parameter $b$ (singular slipping model, $s=1$ ), using $\mathcal{P P S}$ plus regularization, and $\Lambda_{1}=10^{-2}$.

$$
v_{x}=\lambda(h) \frac{\partial v_{x}}{\partial z} \quad(z=0)
$$

where $v_{x}$ is the velocity component parallel to the substrate, $z$ is the normal direction to the plane, $\lambda(h)=\Lambda_{s} / 3 h^{2-s}$ is the slipping length, and $s(<3), \Lambda_{s}$ are positive constants. We consider $s=2$ (constant slipping length $\lambda$ ) and $s=1(\lambda$ $\sim 1 / h$, singular slipping). This boundary condition leads to a modified diffusivity $D(h)$ in Eq. (2), namely,

$$
D_{s}(h)=h^{3}+\Lambda_{s} h^{s} .
$$

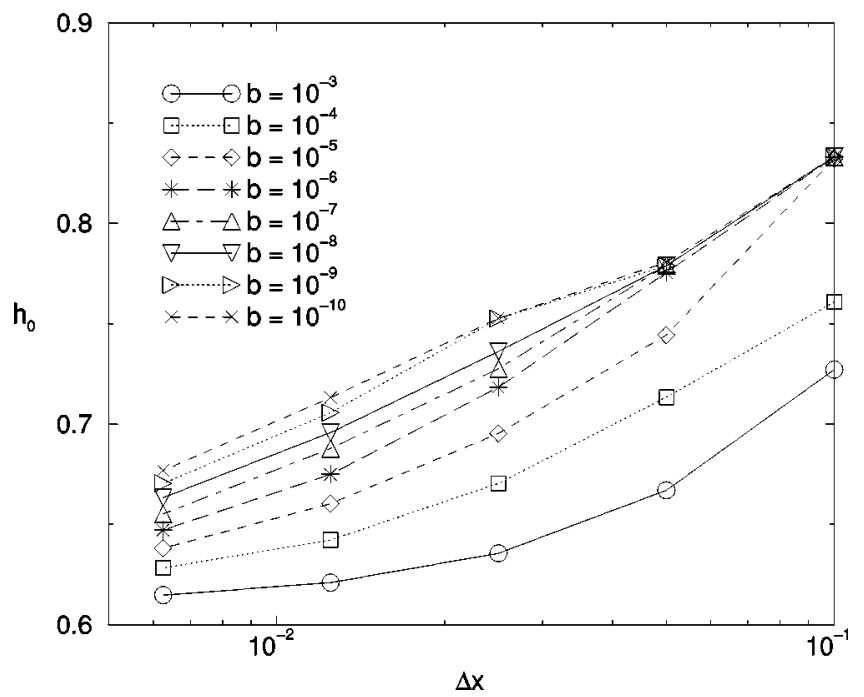

FIG. 10. Thickness $h_{0}$ of the planar drop for $n=3(t=2)$ as a function of $\Delta x$ with several values of the artificial parameter $b$ (constant slipping length model, $s=2$ ), and $\Lambda_{2}=10^{-2}$. PPS is used.
Note that $D_{s}(h) \rightarrow h^{3}$ for $h \gg \Lambda_{s}^{1 /(3-s)}$, i.e., far from the contact line. Also, $D_{s}(h) \rightarrow \Lambda_{s} h^{s}$ as $h \rightarrow 0$, so that moving front solutions are allowed whenever $s<3$.

Now, we compare the behavior of the numerical solution using $s=1$ and $s=2$. In view of the previous test problems (Secs. IV B 1 and IV B 2), both cases are computed using $\mathcal{P P S}$. This involves using $D_{s}(h)$ instead of $D(h)$ in Eqs. (5) and (9), resulting in the following expression for $g(h)(s$ $=1$ requires regularization):

$$
g(h)= \begin{cases}{\left[\ln \left(1+\Lambda_{2} / h\right)-\Lambda_{2} / h\right] / \Lambda_{2}^{2},} & s=2, \\ -\ln \left(1+\Lambda_{1} / h^{2}\right) / 2 \Lambda_{1}-\varepsilon / 3 h^{3}, & s=1 .\end{cases}
$$

Recall that this method also incorporates a lifting by a small amount $b$, that is interpreted as a numerical artifact, not a physical precursor.

Figure 9 shows the central thickness, $h_{0}$, versus $\Delta x$ for $s=1$ with $\Lambda_{1}=10^{-2}$. For small $b$, this thickness becomes $b$-independent, in agreement with [18]. The figure also shows that $\Delta x$ required for convergence does not depend on $b$, provided that $b \lesssim \Lambda_{1}$ (see also Fig. 11). We note that computations with smaller $\Lambda_{1}$, and fully converged in $b$ and $\Delta x$ show that $h_{0}$ increases as $\Lambda_{1}$ decreases. This is because there is more viscous dissipation for smaller $\Lambda_{1}$, as is the case for smaller $b$ in the precursor film model.

Figure 10 shows the case $s=2$ with $\Lambda_{2}=10^{-2}$. We immediately observe that the results for $h_{0}$ show strong $b$-dependence in this range of $\Delta x$ 's, even for very small $b$ 's; this is in contrast to $s=1$ case. Moreover, the $\Delta x$-convergence gets worse as $b$ becomes smaller $\left(d h_{0} / d \Delta x\right.$ increases as $b \rightarrow 0)$. A comparison between the behavior of both slipping models as a function of $b$ is shown in Fig. 11 for $\Delta x=0.05$ and $\Lambda_{1}=\Lambda_{2}=10^{-2}$. For this $\Delta x$ the solution converges for $s=1$, but it does not converge for $s=2$, similarly to $n=1$ (Fig. 4) and $n=2$ (Fig. 6).

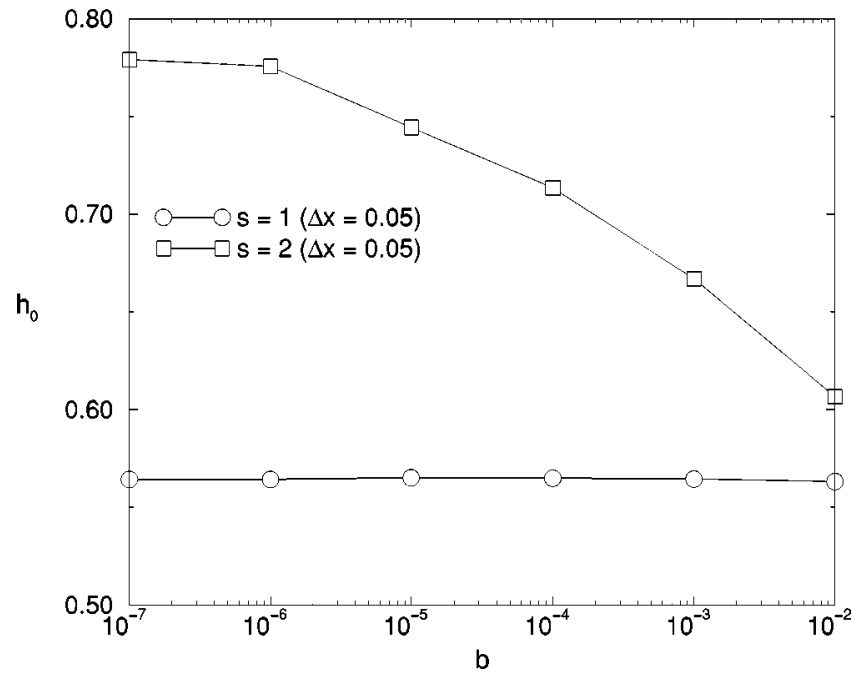

FIG. 11. Thickness $h_{0}$ of the planar drop for $n=3(t=2)$ as a function of the artificial thickness $b$, for the slipping models with $s=1$ and $s=2\left(\Lambda_{1}=\Lambda_{2}=10^{-2}\right)$. PPS is used and regularization is added for $s=1$. 
The difference in the computational performance of the $s=1$ and $s=2$ cases can be understood as follows. Under the slipping models, the diffusivity is given by Eq. (30), and it is required that the term $\Lambda_{s} h^{s}$ is the dominant one as $h \rightarrow 0$. Since we have artificial thickness $b$ at the front, it immediately follows that we must require $b^{3} \ll \Lambda_{s} b^{s}$. This condition gives

$$
\begin{gathered}
b^{2} \ll \Lambda_{1}, \quad s=1, \\
b \ll \Lambda_{2}, \quad s=2
\end{gathered}
$$

and then, the requirement for $s=2$ is much more severe than for $s=1$. This is why $s=2$ needs very small values of $b$ to become $b$-independent.

To summarize, we conclude that a slipping model with $s=2$ (constant slipping length $\lambda$ ) is not viable from the numerical point of view, since it requires very small $\Delta x$ and $b$ for convergence; furthermore, smaller $b$ requires smaller $\Delta x$. Instead, the case $s=1$ seems quite appropriate, since relatively high values of both $b$ and $\Delta x$ yield accurate results. This distinction should be of interest since both models are used in the literature. Related results were reported in [23], where it was shown that computations on relatively coarse grids without slip could lead to very similar results to those obtained by well-resolved computations that assume slip.

\section{B. Long time radial evolution}

Here we present numerical results for the long time evolution of the radial $(\alpha=1)$ drop spreading for $n=3$. We compute the solution with the precursor film and the singular slipping model $(s=1)$. Also, since the $\Delta x$-convergence study was performed above, here we present results only for $\Delta x=0.01$, as a typical case. We do not impose a maximum (small) $\Delta t_{\max }$, but we use a variable time step $\Delta t^{\nu}$, whose value is limited by the requirements of accuracy and positivity, as explained at the end of Sec. III.

The scaling laws obtained in Sec. IV A suggest that we should expect that $x_{f}(t)$ obeys the power law with exponent $\delta=1 / 10$ [see Eq. (19)]. However, since we must introduce some model (precursor film or slipping) to account for the singularity at the front for $n=3$, another condition is added to the problem. This breaks self-similarity, and so we should not expect an exact self similar solution. Our numerics now become a diagnostic to explore deviation from selfsimilarity. We note that a study of the effects of several slipping models on the drop dynamics has been reported in Ref. [22]. Since the description was done by using a quasisteady approach under partially wetting conditions, their results are only qualitatively similar to ours.

Figures 12(a) and 12(b) show $x_{f}(t)$ calculated with the initial condition given by Eqs. (24) and (26), using the precursor and singular slipping models, respectively. The numerical results can be asymptotically fitted by a power law, except for the cases $b=10^{-2}$ for the precursor model, and $\Lambda_{1}=10^{-4}$ for the singular slipping model (artificial $b$ $=10^{-2} \Lambda_{1}$; see Sec. V A 2). We note that the same exponent $\delta \cong 0.11$ is a best fit for power laws for both models. The prefactor $q$ (close to unity) depends on $b$ and $\Lambda_{1}$ in the cor-

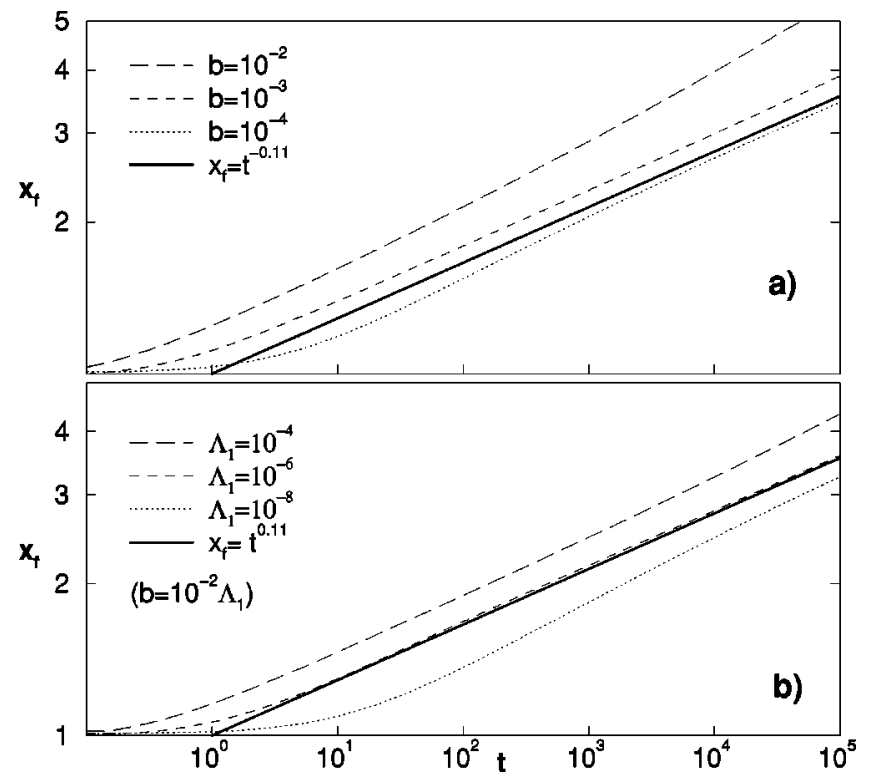

FIG. 12. Front position $x_{f}(t)$ for the radial drop spreading ( $n$ $=3, \alpha=1$ ) under the action of surface tension forces using (a) precursor film model, and (b) singular slipping model $(s=1)$. The line $x_{f}=t^{0.11}$ approximates the asymptotic behavior, as explained in the text.

responding model. Also, the curves $h_{0}(t)$ (not reported here for brevity) consistently tend to a power law with exponent $\beta=-0.22$.

Note that both the precursor film model and the slipping models affect a region of size, say, $\ell$, close to the front, thus introducing a new characteristic length in the problem. This characteristic length is given by $b$ for the precursor film and by $\sqrt{\Lambda_{1}}$ for singular slipping. For the time range shown in Fig. 12 we observe a departure from the self-similar behavior for $\ell \approx b=\sqrt{\Lambda_{1}} \geqslant 10^{-2}$. Additional computations show that this departure is not due to grid effects, and also that it is shifted towards longer times as $\ell$ is decreased. We conjecture that the departure occurs due to the increased ratio of $\ell / h_{0}(t)$ as time progresses; thus, at some time, which depends on $\ell$, introduction of the characteristic length in the model affects the asymptotic (long time) self-similar solution. From our numerical results, we find that this departure starts when $h_{0}(t)$ is about an order of magnitude greater than $\ell$.

The number of time steps $\mathcal{N}$ needed to reach a final time $\tilde{t}$ is a measure of the computational efficiency of each model. We find that for the required accuracy $\left(E^{m}=10^{-2}\right)$, the number of Newton iterations is always small $(3-4)$, independently of the scheme for the interpolation of the diffusivity or the contact line model. Figure 13 shows $\mathcal{N}$ versus $b^{2}$ for the precursor film model, and versus $\Lambda_{1}$ for the singular slipping model. Clearly, the precursor model is much more efficient than the singular slipping model, since for small $\ell$ it requires almost an order of magnitude less time steps. In order to understand why a smaller average time step, $\langle\Delta t\rangle$ $=\tilde{t} / \mathcal{N}$, is required by the slipping model, we performed additional simulations for different combinations of $\Lambda_{1}$ and $b$. We found that, while $\langle\Delta t\rangle$ does not strongly depend on $\Lambda_{1}$, 


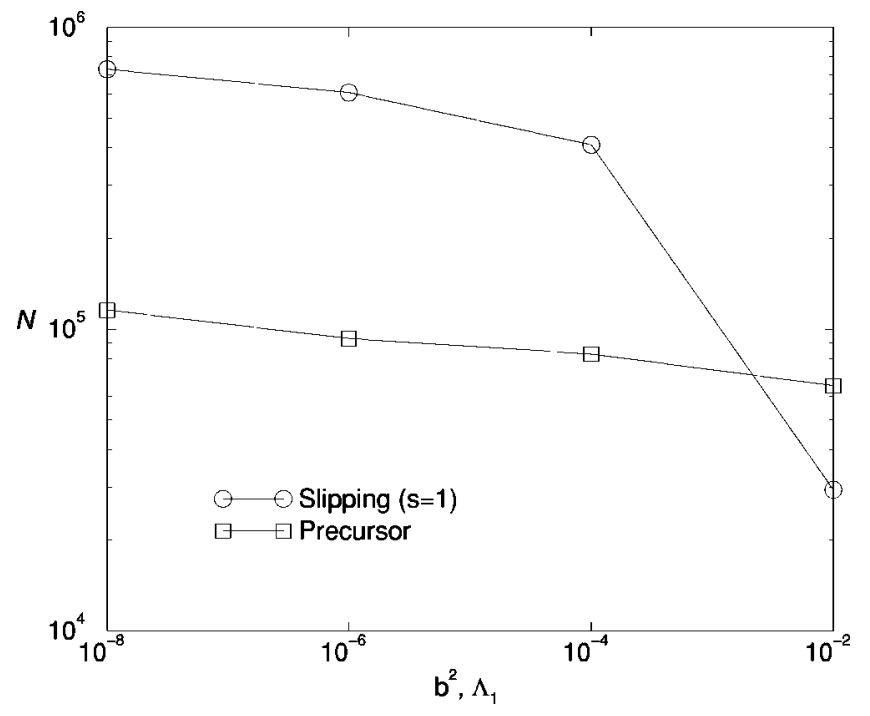

FIG. 13. Number of time steps performed in the simulations of Fig. 12 to reach $t=10^{5}$ as a function of the respective parameter of the model ( $b^{2}$ for the precursor film model, and $\Lambda_{1}$ for the singular slipping model).

it does decrease for smaller $b$. So, it is because of the smallness of $b$ in the slipping model that a very small $\langle\Delta t\rangle$ is required for a given accuracy.

In our simulations we observed that the accuracy condition (see Sec. III) is the main limiting factor of $\Delta t^{\nu}$. Note that $\langle\Delta t\rangle$ can even be of the order of unity (see Fig. 13), due to the fact that the solution is almost self-similar.

\section{GRAVITATIONAL FORCES}

After formulating reliable computational methods for the problems where capillarity is the only driving force, we now consider the gravitational force. Within the framework of lubrication approximation, the gravity effects are modeled by the second order term included in Eq. (1), where $x, h$, and $t$ are scaled by $x_{c}, h_{c}$, and

$$
t_{c}=\frac{3 \mu x_{c}^{4}}{\gamma h_{c}^{3}},
$$

respectively, where $\mu$ is the viscosity and $\gamma$ the surface tension. Consequently, the Bond number is given by $\mathcal{G}$ $=\left(x_{c} / a\right)^{2}$, where $a=\sqrt{\gamma / \rho g}$ is the capillary length, $g$ the gravity and $\rho$ the density.

The gravitational term in Eq. (1) is discretized using standard centered finite differences. We have verified that various conservative discretizations of this terms have comparable performance and efficiency. When using a slipping model instead of the precursor film approach, the replacement $h^{3} \rightarrow h^{3}+\Lambda h^{s}$ must be done in $D(h)$ in Eq. (1), and the corresponding discretization is done in a similar way as described for the precursor film model.

In the preceding section we addressed the problem of a radially spreading drop without gravity, governed by capillary forces. However, it is known that for late times, the gravitational effects become dominant. Consequently, there must be a transition between these two asymptotic regimes. In this section we address this issue by solving directly the governing PDE (1).

We start the calculations with a drop profile defined by Eqs. (24) and (26). The scales $x_{c}$ and $h_{c}$ are given by the respective dimensional initial radius and thickness. Consequently, the dimensional drop volume is given by $V_{c}$ $=(\pi / 6) h_{c} x_{c}^{2}$, which is the only controllable parameter in a laboratory experiment. Thus, we determine $h_{c}$ (consequently, $t_{c}$ ) from Eq. (33), for a given volume, as $h_{c}=6 V_{c} /\left(\pi x_{c}^{2}\right)$, and choose $x_{c}$ by varying $\mathcal{G}$ through the definition $\mathcal{G}$ $=\left(x_{c} / a\right)^{2}$.

Let us first recall the simpler problem of the drop spreading without surface tension but with gravity, i.e., Eq. (1) without the fourth order term and $D(h)=h^{3}$. It is known $[47,48]$ that this second order degenerate PDE admits a selfsimilar solution, called Barenblatt solution. In our dimensionless variables, it reads as

$$
\begin{gathered}
x_{f}=0.894 \ldots\left(\frac{\pi}{6}\right)^{3 / 8}(\mathcal{G} t)^{1 / 8}=0.7014 \ldots(\mathcal{G} t)^{1 / 8}, \\
h(x, t)=\left[1-\left(\frac{x}{x_{f}}\right)^{2}\right]^{1 / 3} .
\end{gathered}
$$

If gravity is the only driving force, this solution gives the asymptotic flow for any given initial condition. When surface tension is also taken into account, the relation between the (global) gravitational and capillary forces is given by the Bond number, $\left(x_{f} / x_{c}\right)^{2}$. For $x_{f} \gg a$ one expects that Barenblatt's solution be asymptotically approached. However, even for large $x_{f}$ surface tension effects may still be relevant in the neighborhood of the contact line. In this (asymptotic) gravity dominated regime one does not expect modification of the exponent $\delta=1 / 8$ due to the presence of localized contact forces, because $\delta$ is a direct consequence of volume conservation (a global condition). Instead, changes in the prefactor of Eq. (34) as well as in the thickness profile, Eq. (35) could be expected. Experiments that show this type of effects have been reported elsewhere [49].

Figure 14 shows the numerical results (dashed lines) for $x_{f}$ using the precursor film model with $\mathcal{G}=1$ in Eq. (1), and using $\mathcal{P P S}$ for the fourth order term. For small $b\left(\leqslant 10^{-4}\right)$ it is possible to obtain an asymptotic behavior with exponent $\delta=1 / 8$, as in Barenblatt's solution [50]. However, the prefactor of the asymptotic line for the numerical results is quite close to unity (the line $x_{f}=t^{1 / 8}$ is shown for comparison), so it is larger than the one of Barenblatt's solution. Note also that the transition to the gravity dominated regime depends strongly on $b$. The smaller $b$, the longer it takes to reach the asymptotic power law behavior.

A comparison with experiments is also shown in Fig. 14. The open and filled symbols correspond to the (nondimensional) experimental data reported in Refs. [43] and [51], respectively, for different volumes $V_{c}$. Note that time is in units of $t_{c}$, Eq. (33), with $x_{c}=a(\mathcal{G}=1)$. Figure 14 shows that a good agreement for small volume drops $\left(V_{c}\right.$ $<2 \mathrm{~mm}^{3}$ ) can be obtained for $b=10^{-5}$, and that smaller 


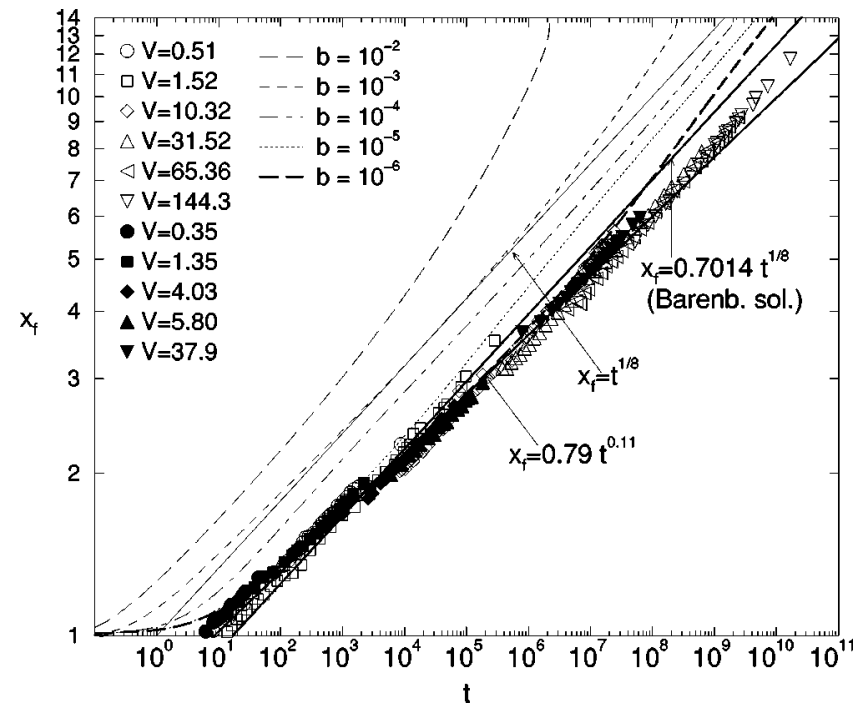

FIG. 14. Front position $x_{f}(t)$ for the radial drop spreading ( $n$ $=3, \alpha=1$ ) under the action of both surface tension and gravity forces for $\mathcal{G}=1$, using precursor film model. The broken lines show numerical results, the open and filled symbols are the experimental data from Refs. [43] and [51], respectively, where the drop volumes are in $\mathrm{mm}^{3}$. The line with the prefactor 0.79 is the best fit to the early times evolution dominated by surface tension.

$b$ 's are required for larger $V_{c}$ [50]. Since $b=h_{p} / h_{c}, h_{p}$ being the dimensional thickness of the actual precursor film, this suggests that in the experiments it must be $h_{p} \approx 800 \AA$ for a typical volume of $10 \mathrm{~mm}^{3}$. Also, it appears that the experimental data for large volume drops tend to approach the gravitational asymptotic line for very long times, in a similar fashion to that of the numerical curves. We note that the dimensional drop volume, $V_{c}$, only specifies the scale for the numerical results, so that the dimensionless computational results shown in Figs. 14 and 15 are volumeindependent. Thus, the data from a given experiment (i.e, a given $V_{c}$ ) fall on a certain range of $x_{f}$ and $t$, in units of $x_{c}$ and $t_{c}$, respectively.

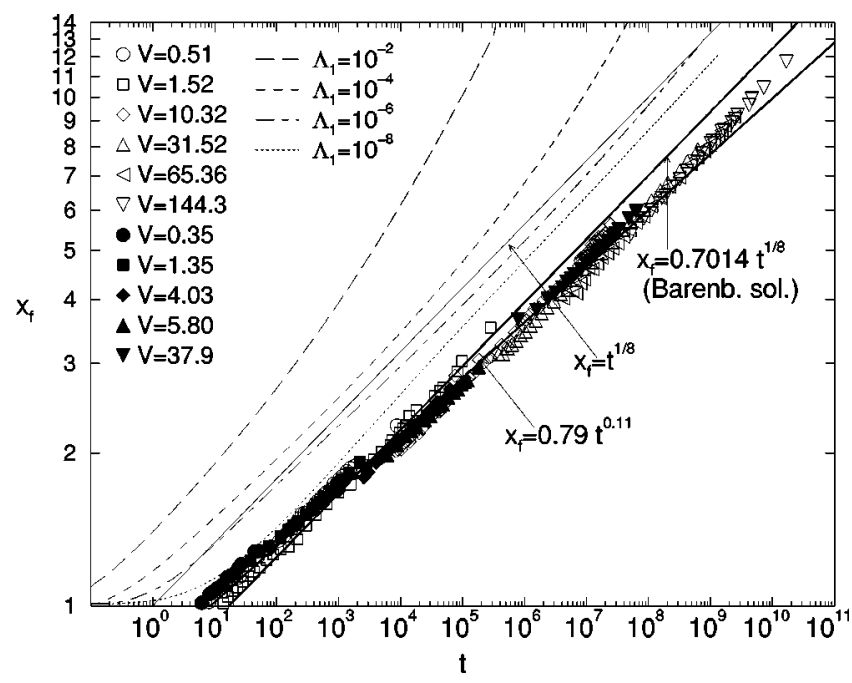

FIG. 15. Front position $x_{f}(t)$ for the radial drop spreading as in Fig. 14, using singular slipping model $(s=1)$.

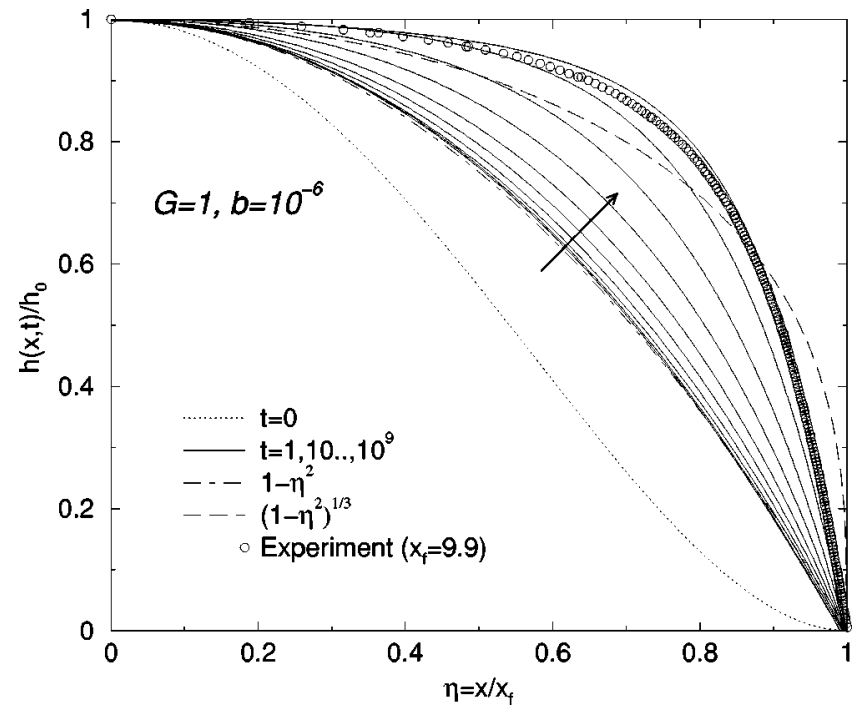

FIG. 16. Profile evolution of a radially spreading drop with both surface tension and gravitational forces present. The arrow shows the direction of time increase. In the simulations we used the precursor film model with $b=10^{-6}$ on a grid size $\Delta x=0.01$ (the profiles are almost insensitive to variation of $\Delta x$ ). The symbols are experimental data from Ref. [43].

Figure 15 shows the results for the singular slipping model (dashed lines). The power law behavior characterized by $\delta=1 / 8$ is observed only for very small $\Lambda_{1}\left(\leqslant 10^{-6}\right)$ and, consequently, for very small artificial $b$. Also, $\Lambda_{1}<10^{-8}$ is required for a good agreement with the experimental data. For smaller $\Lambda_{1}$, a smaller average time step is required; e.g., $\langle\Delta t\rangle$ for $\Lambda_{1}=10^{-6}$ is approximately 8 times smaller than for $\Lambda_{1}=10^{-2}$. Thus, a numerical calculation with the singular slipping model is much less efficient than with the precursor film model.

The increasing effects of gravity as the drop spreads out can also be put in evidence by looking at the change of shape of the thickness profile. In Fig. 16 we plot profiles $h(x, t) / h(0, t)$ vs. $\eta=x / x_{f}(t)$ using precursor film model with $b=10^{-6}$ at different times of the evolution. The flattening of the central part of the drop is evident. A significant difference between the Barenblatt's profile and the most advanced profile is the slope at the front. The computed solution is characterized by profiles which are smoothened by capillary effects close to the contact line, compared to Barenblatt's profile which has infinite slope there. This difference is related with the different prefactors in the numerical asymptotics and the Barenblatt's solution. The symbols in Fig. 16 correspond to the experimental data reported in Ref. [43] for $x_{f}=9.9$ (Fig. 3.f in that paper). The comparison is very favorable, even though the numerical profile at $t=10^{9}$ has a slightly larger $x_{f}(=10.1)$ compared to the experimental one.

Up to our knowledge, this is the first time that this transition between the regimes dominated by surface tension and gravitational forces has been described by the direct solution of the corresponding PDE (see Ref. [43] for an ODE approach). Concerning computational issues, we note that, analogously to Sec. V B, the precursor film model is computationally more efficient than the singular slipping model. 
For instance, for $b^{2}=\Lambda_{1}=10^{-6}\left(\ell \approx 10^{-3}\right)$, the precursor model allows for $\langle\Delta t\rangle 10$ times larger than the one required by the slipping model. Again, this is due to the very small $b$ 's that must be used in the slipping model.

\section{SUMMARY AND CONCLUSIONS}

By means of a series of numerical simulations we have shown the importance of having a good numerical scheme to calculate flows described by Eq. (2), with the presence of advancing fronts. Even without the singularity at the contact line $(n<3)$ and in planar symmetry, the study of the drop spreading problem with $n=1$ and $n=2$ shows how sensitive is the numerical solution to the interpolation of the nonlinear diffusivity.

We obtain that the numerical solutions with $n=1$ are convergent only if the positivity preserving scheme $(\mathcal{P P S})$ plus regularization are employed (Figs. 3 and 4), and that a standard scheme $(\mathcal{S S})$ for the interpolation of the diffusivity fails. For $n=2, \mathcal{S S}$ and $\mathcal{P P S}$ become coincident for small $\Delta x$ (Fig. 5). However, both schemes show that the computation must be done with very small $\Delta x\left(\leqslant 10^{-3}\right)$, in order to obtain a converged solution as $b \rightarrow 0$ (Fig. 6). We conjecture that it might be possible to find a relation $b(\Delta x)$ such that this limit exists, but that study is left for future work. Similar results are obtained in radial symmetry for $n=2$, though they are not reported here for brevity. We believe that this lack of convergence for $n=2$ might be the cause of the low performance of the calculations when using the slipping model with constant slipping length $(s=2)$ in thin film flows $(n=3)$ (see Sec. V A 2).

For $n=3$, and using the precursor film model, both $\mathcal{S S}$ and $\mathcal{P P S}$ seem appropriate to perform the calculation of the drop spreading. The only (but important) difference is that $\mathcal{P P S}$ converges faster, although it is less accurate than $\mathcal{S S}$ for large $\Delta x$ (Fig. 8). An essential point when using slipping models under these schemes is that the results should be insensitive to the "lifting" parameter $b$. The simulations show that the model with constant slipping length $(s=2)$ requires extremely small values of $b$ to achieve this goal, while the singular slipping model $(s=1)$ is much less demanding (Figs. 9 and 10).

The results for the asymptotic description of the radial drop spreading show that both the precursor film model and the singular slipping model are equally appropriate. However, the former is computationally more efficient, since it allows for a much larger $\langle\Delta t\rangle$ for a similar characteristic length $\ell \approx b^{2} \approx \Lambda_{1}$.

These two models show a similar behavior when gravity is included in the radial drop spreading problem (Figs. 14 and 15). Also in this case, the numerical solutions give the correct asymptotic power law behavior with exponent $\delta$ $=1 / 8$, if $\ell$ is sufficiently small. For relatively large volumes $\left(V_{c}>1 \mathrm{~mm}^{3}\right)$, the simulations of Eq. (1) yield results in good agreement with experimental data reported elsewhere [43,51], provided $b \leqslant 10^{-4}$ in the precursor film model and $\Lambda_{1}<10^{-8}$ in the singular slipping model. The simulations also show the transition from the surface tension dominated regime to the gravity dominated regime. Another feature of the gravity effects is the flattening of the profile (Fig. 16), and the finite slope at the front. The latter effect is related to the different values of the prefactor in the power law for $x_{f}(t)$ of the asymptotic gravitational regime and of the Barenblatt's solution.

In summary, since the efficiency is essential for successful modeling of two-dimensional problems, we conclude that the use of the precursor film model is highly advisable. The extension of our computational method to these problems is currently in progress.

\section{ACKNOWLEDGMENTS}

This work was supported by ONR Grant No. N00014-961-0656. J.D. also acknowledges support from Consejo Nacional de Investigaciones Científicas y Técnicas (CONICETArgentina), and Organization of American States (OAS) for his stay at Duke University. The additional support comes from The Sloan Foundation (A.B., L.K.), NSF Grants No. DMR-9321792 and DMS95-04577, and NJIT Grant No. 421210 (L.K.).
[1] E.B. Dussan V., Annu. Rev. Fluid Mech. 11, 317 (1979).

[2] P.G. de Gennes, Rev. Mod. Phys. 57, 827 (1985).

[3] A. Oron, S.H. Davis, and S. Bankoff, Rev. Mod. Phys. 69, 931 (1997).

[4] S.M. Troian, E. Herbolzheimer, S.A. Safran, and J.F. Joanny, Europhys. Lett. 10, 25 (1989).

[5] A.L. Bertozzi and M.P. Brenner, Phys. Fluids 9, 530 (1997).

[6] P. Constantin, T.F. Duppont, R.E. Goldstein, L.P. Kadanoff, M.J. Shelley, and S.M. Zhou, Phys. Rev. E 47, 4169 (1993).

[7] J.R. King, Ph.D. thesis, Oxford University, 1987 (unpublished).

[8] F. Bernis, in Nonlinear Diffusion Equations and their Equilibrium States, edited by N.G. Lloyd, W.-M. Ni, and L.A. Peletier (Springer-Verlag, Basel, 1992), Vol. 3, p. 77.

[9] C.M. Elliott and H. Garcke, SIAM (Soc. Ind. Appl. Math.) J. Math. Anal. 27, 404 (1996).
[10] M.A. Lewis, Theor. Popul. Biol. 45, 277 (1994).

[11] G. Grün, Z. Anal Anwendungen 14, 541 (1995).

[12] S. Boatto, L.P. Kadanoff, and P. Olla, Phys. Rev. E 48, 4423 (1993).

[13] V. Ludviksson and E.N. Lightfoot, AIChE J. 14, 674 (1968).

[14] R.J. Hansen and T.Y. Toong, J. Colloid Interface Sci. 36, 410 (1971).

[15] M.H. Eres, L.W. Schwartz, and R.V. Roy, Phys. Fluids 12, 1278 (2000).

[16] J. Lowengrub and L. Truskinovsky, Proc. R. Soc. London, Ser. A 454, 2617 (1998).

[17] P.H. Leo, J.S. Lowengrub, and J.H. Jou, Acta Mater. 46, 2113 (1998).

[18] E.B. Dussan V., J. Fluid Mech. 77, 665 (1976).

[19] H.P. Greenspan, J. Fluid Mech. 84, 125 (1978). 
[20] H.P. Greenspan and B.M. McCay, Stud. Appl. Math. 64, 95 (1981).

[21] L.M. Hocking and A.D. Rivers, J. Fluid Mech. 121, 425 (1982)

[22] P.J. Haley and M.J. Miksis, J. Fluid Mech. 223, 57 (1991).

[23] J.A. Moriarty and L.W. Schwartz, J. Eng. Math. 26, 81 (1992).

[24] A.L. Bertozzi, Notices Am. Math. Soc. 45, 689 (1998).

[25] L. Zhornitskaya and A.L. Bertozzi, SIAM (Soc. Ind. Appl. Math.) J. Numer. Anal. 37, 523 (2000).

[26] J.W. Barrett, J.F. Blowey, and H. Garcke, Numer. Math. 80, 525 (1998).

[27] G. Grün and Rumpf (unpublished).

[28] An example of a weak form of Eq. (2) satisfies $\int h(\partial \psi / \partial t)$ $=\int \boldsymbol{\nabla}^{2} h\left[D(h) \boldsymbol{\nabla}^{2} \psi+D(h) \boldsymbol{\nabla} h \cdot \boldsymbol{\nabla} \psi\right] d x d t$ for any sufficiently smooth test function $\psi(x, t)$ where the integrals are over all time and space. This expression is obtained by multiplying Eq. (2) by $\psi$, and integrating twice by parts with no-flow boundary conditions, $\boldsymbol{\nabla} h=\boldsymbol{\nabla} c=0$ (other similar definitions may be found in the literature). Such formulations allow for solutions of the equation with discontinuous derivatives (as in the case of a moving contact line) where the solution goes to zero.

[29] R.E. Goldstein, A.I. Pesci, and M.J. Shelley, Phys. Rev. Lett. 70, 3043 (1993).

[30] R.E. Goldstein, A.I. Pesci, and M.J. Shelley, Phys. Rev. Lett. 75, 3665 (1995).

[31] F. Bernis and A. Friedman, J. Diff. Eqns. 83, 179 (1990).

[32] A.L. Bertozzi, M.P. Brenner, T.F. Dupont, and L.P. Kadanoff, in Trends and Perspectives in Applied Mathematics, edited by L. Sirovich, Applied Mathematical Sciences Vol. 100 (Springer-Verlag, New York, 1994), p. 155.

[33] E. Beretta, M. Berstch, and R. Dal Passo, Arch. Ration. Mech. Anal. 129, 175 (1995).
[34] A.L. Bertozzi and M. Pugh, Commun. Pure Appl. Math. 49, 85 (1996).

[35] T.F. Dupont, R.E. Goldstein, L.P. Kadanoff, and S. Zhou, Phys. Rev. E 47, 4182 (1993).

[36] A.L. Bertozzi, SIAM (Soc. Ind. Appl. Math.) J. Appl. Math. 56, 681 (1996).

[37] R. Almgren, A.L. Bertozzi, and M.P. Brenner, Phys. Fluids 8, 1356 (1996).

[38] L. Zhornitskaya, Ph.D. thesis, Duke University, 1999 (unpublished).

[39] F. Bernis, L.A. Peletier, and S.M. Williams, Nonlinear Analysis, Theory, Methods and Applications 18, 217 (1992).

[40] R. Ferreira and F. Bernis, Eur. J. Appl. Math. 8, 507 (1997).

[41] G.I. Barenblatt, Scaling, Self-Similarity, and Intermediate Asymptotics (Cambridge University Press, New York, 1996).

[42] M. Brenner and A.L. Bertozzi, Phys. Rev. Lett. 71, 593 (1993).

[43] R. Gratton, J. Diez, L. Thomas, B. Marino, and S. Betelú, Phys. Rev. E 53, 3563 (1996).

[44] P. Ehrhard and S. Davis, J. Fluid Mech. 257, 463 (1993).

[45] V.V. Kalinin and V.M. Starov, Colloid J. Russian Acad. Sci. 50, 19 (1988).

[46] W.D. Bascom, R.L. Cottington, and C.R. Singleterry, Adv. Chem. Am. Chem. Soc. 43, 389 (1964).

[47] H.E. Huppert, J. Fluid Mech. 121, 43 (1982).

[48] J. Gratton and F. Minotti, J. Fluid Mech. 210, 155 (1990).

[49] B.M. Marino, L.P. Thomas, J.A. Diez, and R. Gratton, J. Colloid Interface Sci. 177, 14 (1996).

[50] Calculations with such small $b$ 's are not computationally too expensive since, for instance, the average time step $\langle\Delta t\rangle$ for $b=10^{-4}$ is approximately only $30 \%$ smaller than for $b$ $=10^{-2}$.

[51] A.M. Cazabat and M.A. Cohen Stuart, J. Phys. Chem. 90, 5845 (1986). 